How to reference this article Miszalska, J. (2019). Società letteraria delle donne tra Italia e Polonia. Italica Wratislaviensia, 10(2), 325-334.

DOI: http://dx.doi.org/10.15804/IW.2019.10.1.32

Jadwiga Miszalska

Uniwersytet Jagielloński w Krakowie jadwiga.miszalska@uj.edu.pl

ORCID: 0000-0001-7122-9396

\title{
SOCIETÀ LETTERARIA DELLE DONNE TRA ITALIA E POLONIA
}

Anita Kłos (2018). Apologia kobiecego ducha. Sibilla Aleramo i jej zwiazki z polska kultura literacka pierwszej połowy XX wieku. Lublin: Wydawnictwo Uniwersytetu Marii Curie-Skłodowskiej, pp. 292.

1

ormai da quasi cinque decenni, cioè da quando apparve la riedizione di Una donna (1973), che l'opera di Sibilla Aleramo, spesso definita come la prima femminista italiana, suscita l'interesse di studiosi e soprattutto studiose italiane. Alcuni anni fa, infatti, un gruppo di ricercatrici della "Sapienza"diretto dalla prof.ssa Marina Zancan aveva ideato un progetto di recupero per la storia e la critica letteraria italiane, dedicato alla produzione di donne scrittrici spesso sottovalutate o addirittura dimenticate. Il progetto doveva avere una solida base documentaria offerta dagli archivi personali delle scrittrici. Uno degli archivi che era risultato una miniera di informazioni preziose, non solo sulla vita della sua protagonista, ma anche sugli ambienti letterari italiani coevi, è quello di Sibilla Aleramo, ora custodito nella sede della Fondazione Gramsci di Roma. Nel 1988 è stato pubblicato il volume Svelamento. Sibilla Aleramo: una biografia intellettuale curato da Annarita Buttafuoco e Marina Zancan che ha dato avvio a ulteriori studi dedicati alla scrittrice. Sibilla 
Aleramo, secondo Marina Zancan caso "esemplare" della scrittura femminile, è divenuta oggetto di studi anche fuori d'Italia, apprezzata tra l'altro da Sharon Wood, autrice della monografia sulla scrittura delle donne in Italia, Italian Women's Writing 1860-1994 (1995).

Anita Kłos, italianista, docente dell'Università di Lublino, da alcuni anni studia la problematica delle relazioni culturali e letterarie tra Italia e Polonia con uno sguardo particolarmente attento all'attività delle donne: da una parte la presenza delle scrittrici italiane in Polonia, dall'altra il ruolo delle donne, traduttrici e letterate polacche nella diffusione della letteratura italiana. Nel corso delle ricerche concernenti le traduzioni ha scoperto la versione polacca di Una donna, pubblicata nel 1909 su una prestigiosa rivista polacca di opinione e di cultura, "Prawda" (La verità). Così è cominciata la sua avventura con Sibilla, un po' da detective. Le giornate passate all'archivio della Fondazione Gramsci hanno infatti portato alla luce documenti finora sconosciuti riguardanti i contatti dell'Aleramo con ambienti letterari polacchi nei primi decenni del Novecento. Il frutto di queste ricerche è il libro che stiamo presentando e il cui titolo allude al titolo del saggio della protagonista del libro, Apologia dello Spirito Femminile pubblicato su "Il Marzocco" nel 1911 (Aleramo, 1911). Il libro di Anita Kłos copre l'arco di una quarantina di anni ed è un tentativo di ricostruire i numerosi contatti che la scrittrice ebbe con diversi rappresentanti della vita letteraria polacca a partire dal primo decennio del Novecento. Infatti questi contatti si svolgono in due tappe: prima della II guerra mondiale quando l'Aleramo è tradotta in Polonia e quando lei stessa traduce una pièce di Zofia Nałkowska, e dopo la guerra con la sua presenza al Congresso Mondiale per la Pace svoltosi a Breslavia nel 1948 da cui scaturiscono conoscenze con letterati polacchi di sinistra.

Il quadro, estremamente complesso, offerto nel libro della Kłos, ricostruisce alcune reti di amicizie e relazioni intercorse tra la scrittrice italiana ed esponenti della cultura polacca. Studiando le traduzioni che costituiscono il punto di partenza, la studiosa, conformemente alle odierne tendenze vigenti nei Translation Studies, va oltre il testo. Le interessa ciò che si svolge intorno ad esso, diversi condizionamenti e circostanze, pressioni politiche, economiche, ovvero quello che fa parte della so- 
ciologia della scrittura e infine la "microstoria" dell'autore/traduttore ricostruibile in base ai documenti personali spesso molto intimi. D'altra parte, trattandosi della scrittura delle donne, l'autrice non perde di vista quello che nei loro testi e nella loro lingua costituisce la testimonianza dello "spirito femminile".

Sibilla Aleramo non è molto conosciuta in Polonia, neanche nelle cerchie degli italianisti: raramente nominata dai compendi di storia della letteratura italiana, ricordata spesso per la sua relazione con Dino Campana, appare come un caso letterario solo in poche pubblicazioni più recenti. Per cui il libro della Kłos si apre giustamente con un capitolo introduttivo che presenta al lettore polacco la figura della scrittrice. Non è facile presentare in poche pagine l'opera di un personaggio così complesso come l'Aleramo, l'autrice tuttavia ci riesce benissimo, delineando nella sua "biografia intellettuale" i punti cardine e le linee di sviluppo della produzione letteraria, basandosi sui suoi appunti personali ritrovati tra le carte dell'archivio: il che permette di assumere una particolare prospettiva, come se la scrittrice stessa raccontasse la propria vita e la ricerca della propria identità letteraria e umana.

La parte biografica prepara quello che costituisce il fulcro del lavoro. Le due parti successive presentano l'Aleramo in duplice veste: come autrice tradotta e come traduttrice. La prima ha come tema la traduzione polacca di Una donna. Nel Fondo Aleramo si è conservata la corrispondenza della scrittrice con la traduttrice Soava Gallone, che ha costituito il punto di partenza per la ricostruzione delle vicende polacche del libro. Anita Kłos grazie alla minuziosa ricerca delle informazioni riguardanti le protagoniste di questa storia, condotta in diversi archivi e sulla stampa riesce a fornire un quadro alquanto completo delle relazioni tra le due donne. Innanzitutto scopre l'identità della traduttrice che risulta un personaggio non meno affascinante della scrittrice italiana. Soava (Stanisława) Gallone era un'ebrea polacca, nata in una benestante famiglia di industriali e intellettuali. Grazie alla madre, medica laureatasi a Sorbonne, anche Soava ha ricevuto una buona istruzione. Studiò a Parigi, medicina, filologia o arti teatrali. Nel 1907 la donna già sposata per la prima volta arrivò in Italia dove a Sorrento conobbe il suo secondo marito, il regista Carmine Gallone. Questa storia romantica cambiò la 
sua vita. Oltre a continuare i suoi interessi legati alla letteratura, divenne in poco tempo una delle più famose dive del cinema muto, collaborando in seguito con suo marito nell'ambito dell'industria cinematografica. Soava e Sibilla frequentavano negli anni 1907-1908 gli stessi ambienti artistici a Sorrento e a Roma, ma non si conoscevano di persona, quando Soava avanzò la proposta di tradurre Una donna, la cui lettura le era stata suggerita dal marito. Angelo Flavio Guidi, scrittore, amico della Gallone e dell'Aleramo, in una lettera del marzo 1908 indirizzata a Sibilla raccomandava Soava come traduttrice, sottolineando le sue ampie competenze letterarie e le conoscenze negli ambienti editoriali di Varsavia. Infatti Soava nei primi anni del suo soggiorno italiano era incline alla carriera giornalistica. Così comincia anche la sua corrispondenza con la scrittrice in cui racconta delle sue, non ancora grandi, esperienze (come ammette lei stessa) traduttive. Infatti a quei tempi la Gallone intraprende diverse traduzioni dall'italiano in polacco e dal polacco in italiano, ma non tutte portate a buon fine. Tra queste il proposito di tradurre Una don$n a$ risulta senz'altro il progetto più ambizioso. Il lavoro sulla traduzione interrotto dalla malattia della traduttrice si conclude finalmente nell'agosto del 1909. La Gallone, approfittando dei suoi contatti varsaviani, cercava una casa editrice propensa a pubblicare il lavoro; in fin dei conti il romanzo esce - con una certa insoddisfazione dell' autrice - a puntate su una rivista. Non si può dire però che questa fosse una sconfitta. Infatti il settimanale "Prawda" era uno dei più prestigiosi periodici polacchi. Di orientamento liberal-socialista e progressista, pubblicava solo opere in conformità con la propria linea ideologica e di un certo livello artistico. In più, non volendo attirare i lettori con metodi di marketing troppo facili, puntava su testi brevi e la concessione accordata per il romanzo dell'Aleramo, ci dice molto. Non soddisfacente dal punto di vista economico né per l'autrice né per la traduttrice, la pubblicazione della Kobieta (Aleramo, 1909, 1910), preceduta da un suo caloroso ritratto preparato dalla traduttrice, fu un evento importante e ad oggi l'unica traduzione polacca della scrittrice.

Avendo presentato le circostanze della nascita della versione polacca del romanzo, Anita Kłos si sofferma sull'analisi della traduzione. Una donna per molti versi è un'opera eccezionale; costituisce una prima 
tappa nella ricerca della lingua femminile liberata da matrici maschili, una lingua che costituisce il perno dell'identità della Donna Nuova. La studiosa identifica le tracce di una liricità tutta femminile soprattutto a livello sintattico e ritmico, ottenuta tramite ripetizioni, giochi fonici e un uso coraggioso dell'interpunzione. La minuziosa analisi proposta da Anita Kłos ci presenta il testo della Gallone come una traduzione "decente", esteticamente soddisfacente, ma che in realtà non riesce a riprodurre "lo spirito" dell'originale. Con procedimenti razionalizzanti secondo la terminologia di Antoine Berman - (1999) a livello di sintassi e interpunzione, con numerose concretizzazioni, con diversa segmentazione del testo, la traduzione perde quello che costituisce per molti versi il perno e il valore di Una donna: la presentazione dell'anima femminile con i suoi moti e passioni tradotti in lingua. Kobieta della Gallone si manifesta soprattutto come un testo autobiografico in cui la cosa più importante è la storia raccontata e in cui - come osserva la studiosa vengono cancellate le tracce della femminilità. Questa interpretazione tuttavia non differiva molto dalla lettura che del romanzo proposero "eminenti critici" italiani richiamati dalla Gallone nella nota sull'autrice. Inoltre Anita Kłos annota a ragione che sarebbe ingiusto dare tutta la colpa dei cambiamenti solo alla traduttrice. Non sappiamo infatti se semplificazioni e tagli effettuati non fossero dovuti all'intervento della redazione. Infatti, pubblicando un romanzo su un periodico, si doveva sempre adattarlo alla poetica del testo "a puntate", secondo la quale ogni pezzo doveva costituire un'entità in un certo senso conclusa e di una lunghezza prestabilita.

A questo punto l'autrice del libro si domanda in che misura il romanzo dell'Aleramo si iscriva nella realtà polacca di allora. Per capirlo bisogna vedere la situazione della donna e dei movimenti femministi nelle due società. Benché la situazione giuridica della donna polacca e italiana non differisse, diversa era la loro situazione sociale dovuta alla differenze culturali e storiche delle due nazioni. La donna polacca, come osserva anche la Gallone, godeva di una maggiore libertà nella vita di ogni giorno, il problema dell'emancipazione, tuttavia occupava le menti femminili in ambedue i paesi. La stessa Gallone può essere annoverata tra le femministe polacche e la Kłos cerca di definire quale tipo di fem- 
minismo rappresentino le due letterate. A questo serve anche una breve, ma densa, presentazione del femminismo polacco di cui facevano parte le attiviste per l'emancipazione, le suffragiste, ma anche numerose giornaliste e scrittrici. Nella presentazione che precede la pubblicazione di Kobieta sulla "Prawda", il libro è confrontato con il romanzo, allora fresco di stampa, Dzieje grzechu (Storia del peccato) di Stefan Żeromski, storia di una donna tradita, diventata prostituta e infanticida, raccontata dalla prospettiva della protagonista. La Gallone deve aver notato certi parallelismi tra i due testi, e toccata profondamente dal romanzo polacco, intraprese la sua traduzione in italiano, probabilmente mai portata a compimento; in seguito nel 1918 interpretò la parte della protagonista Eva nel film tratto dal romanzo, girato da suo marito. Di questi parallelismi, differenze, punti comuni tra femminismo sociale e letterario nei due paesi, che qui non possono essere sviluppati, scrive in modo interessantissimo Anita Kłos, costatando infine che mancano tracce di una reale ricezione polacca del romanzo aleramiano. Alla sua attenzione tuttavia non sfugge un particolare importante. Il romanzo fu letto da Zofia Nałkowska, valida scrittrice polacca impegnata nei dibattiti femministi, che ne scrive all'Aleramo in una lettera del 1930. Nałkowska è solidale con Sibilla nella convinzione che nella lotta per la Donna Nuova, sia importante rivendicare il diritto per una "vita completa" anche in senso erotico, il cui raggiungimento è bloccato secondo la scrittrice italiana dall'egoismo maschile e secondo la polacca dalla natura stessa della donna, riluttante ad assumere una piena responsabilità di sé. Le relazioni tra Zofia Nałkowska e Sibilla Aleramo costituiscono l'argomento della terza parte del libro.

Frugando tra le carte del Fondo Aleramo la studiosa polacca ha scoperto che le relazioni con Soava Gallone, la quale ebbe incontri con Sibilla fino agli anni trenta, non esauriscono i contatti della scrittrice con la Polonia. Infatti Nałkowska è un'altra letterata con cui Sibilla entra in relazioni epistolari. Ma la storia comincia grazie alla giornalista, scrittrice e traduttrice Emilia Szenwic con cui l'Aleramo strinse una duratura relazione di amicizia. Emilia Szenwic, moglie di un rinomato avvocato di Varsavia e professore di diritto, vivace letterata e giornalista possedeva fin dal 1927 una residenza estiva a Positano, chiamata "Stella 
Romana”. La villa, menzionata dalla Aleramo nel romanzo Il frustino e nella poesia Positano, presto divenne luogo di ritrovo di numerosi intellettuali, letterati e artisti, immigrati russi e tedeschi, villeggianti polacchi e ovviamente anche italiani. Qui spesso d'estate soleva riposare anche l'Aleramo. La Szenwic si dimostrò una grande ammiratrice della sua scrittura e conoscitrice della cultura italiana, pubblicando sulle più prestigiose riviste polacche articoli sulle tematiche italiane. Dopo la morte del marito avvenuta nel 1936 la scrittrice vendette l'appartamento varsaviano trasferendosi in Italia, dove rimase fino alla morte non tornando più in patria. Nelle dense pagine del libro della Kłos ritroveremo la fitta rete dei contatti della Szenwic e l'elenco dei suoi lavori: traduzioni, articoli, ma la ricerca nell'archivio porta alla luce una cosa molto più preziosa: la storia della amicizia, delle relazioni umane, che si sono create tra la proprietaria della villa e i suoi ospiti, tra i quali la protagonista del libro occupò un posto privilegiato. Dalle lettere veniamo a sapere che la Szenwic aveva in mente alcuni progetti editoriali. Cercava senza successo editori e traduttori polacchi per i testi dell'Aleramo che ammirava tanto. Malgrado i tentativi di far interessare alla produzione aleramiana importanti esponenti della vita letteraria polacca: Zofia Nałkowska, Zuzanna Rabska, Julia Dickstein-Wieleżyńska, Kobieta rimarrà l'unico testo tradotto in polacco. Il merito della Szenwic è tuttavia l'introduzione del nome dell'Aleramo nella coscienza letteraria polacca. Oltre ai suoi articoli, negli anni trenta apparvero alcuni testi di altri critici polacchi dedicati la scrittrice.

La mediazione della Szenwic tuttavia non era a senso unico. Nel 1930 cominciano le trattative - in cui lei ha un ruolo attivo - per la traduzione del dramma Dom kobiet di Zofia Nałkowska, la cui messa in scena aveva incontrato il plauso del pubblico polacco. Il libro presenta ampiamente le circostanze della nascita del dramma e le svariate reazioni della critica polacca, dall'entusiasmo fino alle stroncature misogine, nonché le possibili linee di interpretazione del testo. La traduzione del dramma stesa dall'Aleramo risulta forse il più prezioso ritrovamento fatto dalla Kłos nell'archivio della Fondazione Gramsci: il testo, infatti, non era noto agli studiosi. La storia del testo, rimasto in forma manoscritta, rimane oscura fino agli anni del dopoguerra. Come risulta dalle carte 
dell'archivio, l'Aleramo tentò di far portare la pièce sul palcoscenico, rivolgendosi a rinomati registi, senza però un esito positivo. Solo nel 2018, quasi novant'anni dopo la prima stesura, il libro ha visto la luce, curato e munito di un'ampia introduzione da Anita Kłos, nella collana "Parole diverse", dedicata alla scrittura delle donne (Zofia Nałkowska, La casa delle donne, Pacini Editore 2018).

Non conoscendo il polacco, la scrittrice nel suo lavoro approfittò dalla traduzione filologica di una certa Maria Poznańska, nonché dalla traduzione francese di Thérèse Koerner. Anita Kłos analizza il testo da due punti di vista. Cerca di contestualizzarlo nell' ambito della produzione aleramiana, innanzitutto nel contesto della sua scrittura teatrale per cui non prescindendo dagli aspetti ideologici e da una certa comunanza delle anime (interessante la trama della pièce con protagoniste solo donne, fortemente condizionate dalla "assente presenza" dei maschi), si sofferma anche su quelli prettamente estetici. D'altra parte - considerando i lavori traduttivi di Sibilla - studia il testo in quanto traduzione, tanto più complessa perché indiretta e al tempo stesso interlinguistica e intralinguistica. Le due versioni di cui dispone Aleramo hanno un carattere completamente diverso, se non opposto. La traduzione della Poznańska ha un carattere "letterario" ed è decisamente una traduzione straniante che conserva le tracce della cultura della fonte. Quella della Koerner, preparata appositamente per la scena, è attenta alla performatività del linguaggio ed elimina i tratti culturali specifici mirando alla domesticazione. La traduttrice francese rinuncia anche a molti dettagli, peculiarità degli oggetti, tracce degli idioletti dei personaggi che contribuiscono alla loro caratteristica e creano una specie di polifonismo, badando invece solo alle informazioni che spingono l'azione avanti.Aleramo, secondo la Kłos, si situa in una posizione di mezzo, prendendo come base la versione della Koerner, depurata da elementi culturali e richiami al culto cattolico, ma completandola con alcuni frammenti della Poznańska, che considera stilisticamente riusciti e interessanti dal punto di vista letterario. Confrontando La casa delle donne con altre traduzioni eseguite dall'Aleramo, la Kłos ci offre un primo intervento sull'Aleramo-traduttrice, finora trascurata dalla critica. 
Anita Kłos ci propone un libro affascinante, in quanto sa unire nel volume un solido lavoro scientifico con il racconto delle storie personali, intime delle protagoniste della cultura italiana e polacca, dando al testo una vivacità da reportage. Il discorso è costruito su una ben salda base metodologica, con il richiamo di una ricchissima bibliografia teorica che rimanda a studi traduttologici, letterari e culturali. Un frammento di storia delle relazioni letterarie italo-polacche viene raccontato prendendo come punto di partenza dei fili di amicizie e contatti interpersonali in modo tale che questa storia assume il volto di persone concrete, donne scrittrici, per poi passare all'ampio contesto della cultura polacca, italiana e addirittura europea, il tutto reso possibile grazie a un paziente lavoro archeologico negli archivi e a minuziose ricerche bibliografiche. Il libro di Anita Kłos è una vera miniera di informazioni concernenti l'attività delle donne intellettuali, italiane e polacche nell'arco di una quarantina di anni. Oltre ai nomi delle due protagoniste -Aleramo e Nałkowska - si tratta di donne in parte o completamente dimenticate, la cui presenza attiva in diversi campi della cultura può meravigliare. L'autrice tratteggia un quadro di una società di donne, appartenenti a culture diverse, ma legate da fili di comprensione nella loro lotta per la parità dei diritti. Il punto centrale di queste considerazioni è il tema della traduzione come creatrice di culture: traduzione, che spesso in modo stereotipato, ma avente la sua conferma nella prassi, viene associata all'attività delle donne. Per concludere questa - per necessità non esauriente presentazione - mi resta da aggiungere che la lettura del libro di Anita Kłos è un vero piacere, anche per la bellezza e precisione del linguaggio in cui è scritto.

\section{BIBLIOGRAFIA}

Aleramo, S. (1911). Apologia dello Spirito Femminile. Il Marzocco. n. 15, 4. Aleramo, S. $(1909,1910)$ Kobieta. Trad. S. Gallone. Prawda n. 35, 37-41, 43, 45-46, 48-49; 1-23.

Berman, A. (1999). La traduction et la lettre ou l'Auberge du lointain, Paris: Éditions du Seuil. 
Buttafuoco, A., Zancan, M. (a cura di). (1988). Svelamento. Sibilla Aleramo: una biografia intellettuale. Milano: Feltrinelli.

Wood, S. (1995). Italian Women's Writring 1860-1994. London: The Athlon Press.

Nałkowska, Z. (2018). La casa delle donne. Trad. S. Aleramo. Pisa: Pacini Editore. 